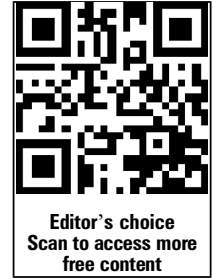

Department of Adult Congenital Heart Disease, Royal Brompton Hospital, London, UK

${ }^{2}$ NIHR Cardiovascular Biomedical Research Unit, Royal Brompton \& Harefield NHS Foundation Trust and Imperial College, London, UK ${ }^{3}$ East Midlands Congenital Heart Centre, Glenfield Hospital, University Hospitals of Leicester, Leicester, UK and Cardiovascular Biomedical Research Unit, University of Leicester

${ }^{4}$ Cardiology Department, Patras University Hospital, Patras, Greece

${ }^{5}$ Department of Haematology, Royal Brompton Hospital, London, UK

\section{Correspondence to} Dr Sonya V Babu-Narayan, Department of Adult Congenital Heart Disease, National Heart and Lung Institute, Imperial College London, Royal Brompton Hospital, Sydney Street, London SW3 6NP, UK; s.babu-narayan@rbht.nhs.uk

Received 23 June 2014 Revised 29 August 2014 Accepted 29 September 2014 Published Online First 28 October 2014

\section{SLinked}

- http://dx.doi.org/10.1136/ heartjnl-2014-306897

\section{CrossMark}

To cite: Heng EL, Bolger AP, Kempny A, et al. Heart 2015;101:447-454.

\title{
Neurohormonal activation and its relation to outcomes late after repair of tetralogy of Fallot
}

\author{
Ee Ling Heng, ${ }^{1,2}$ Aidan P Bolger, ${ }^{3}$ Alexander Kempny, ${ }^{1}$ Periklis A Davlouros, ${ }^{4}$ \\ Simon Davidson, ${ }^{5}$ Lorna Swan, ${ }^{1}$ Anselm Uebing, ${ }^{1}$ Dudley J Pennell, ${ }^{2}$ \\ Michael A Gatzoulis, ${ }^{1,2}$ Sonya V Babu-Narayan ${ }^{1,2}$
}

\section{ABSTRACT \\ Background Brain natriuretic peptide (BNP) levels are elevated in patients with repaired Tetralogy of Fallot (rTOF), the clinical significance of which remains uncertain.}

Methods and results Ninety consecutive adults ( $\geq 16$ years) with rTOF (mean age $32.7 \pm 11.3$ years, $64 \%$ men) were prospectively recruited from a single tertiary centre, together with 15 age-matched and gendermatched controls. Patients with rTOF had elevated BNP (8.9 (5.9-14.6) vs $5.4(2.2-7.5) \mathrm{pmol} / \mathrm{L} ; \mathrm{p}<0.01)$, and BNP activation was common even in asymptomatic patients. Also, atrial natriuretic peptide $(6.9(4.0-9.9)$ vs $3.3(1.0-4.0) \mathrm{pmol} / \mathrm{L} ; \mathrm{p}<0.01)$, endothelin-1 (1.14 $(0.94-1.48)$ vs $0.75(0.44-0.93) \mathrm{pmol} / \mathrm{L} ; \mathrm{p}<0.01)$ and renin (55.0 (45.5-66.5) vs $18.6(12.0-22.7) \mathrm{pmol} / \mathrm{L}$; $p<0.01)$ were elevated at baseline compared with controls. Interactions between BNP with endothelin-1, cardiothoracic ratio and right atrial area were evident. Eight deaths occurred over a median follow-up of 10 years. On Cox regression analysis, BNP emerged as a strong predictor of death (HR 1.16 per $10 \mathrm{pmol} / \mathrm{L}, 95 \%$ Cl 1.05 to $1.29 ; p<0.01)$. Survival receiver operating curve analysis revealed an optimum cut-off of BNP $\geq 15 \mathrm{pmol} / \mathrm{L}$ (=52 pg/mL), above which BNP was related to significantly increased mortality (HR $5.40,95 \% \mathrm{Cl}$ 1.29 to $22.6 ; p<0.01)$; absolute mortality at 5 years $19 \%$ vs $3 \%$ in patients with BNP $\leq 15 \mathrm{pmol} / \mathrm{L}$. BNP was also a predictor of sustained arrhythmia (HR 2.06 per $10 \mathrm{pmol} / \mathrm{L}, 95 \% \mathrm{Cl} 1.32$ to $3.21 ; \mathrm{p}<0.05)$.

Conclusions Neurohormonal activation is present in adults with rTOF including asymptomatic patients. BNP level $\geq 15 \mathrm{pmol} / \mathrm{L}$ is associated with a fivefold increased risk of death. These data suggest that BNP measurement in patients with rTOF should be incorporated in the periodic risk stratification assessment of these patients under lifelong follow-up.

\section{INTRODUCTION}

Tetralogy of Fallot is the most common cyanotic congenital heart disease (CHD). It accounts for $10 \%$ of patients with CHD, occurring in 1 in 3600 births. ${ }^{1}$ There is a growing adult population of patients with repaired tetralogy of Fallot (rTOF) requiring lifelong follow-up, with correspondingly increasing numbers of patients at risk of adverse outcomes including ventricular dysfunction, arrhythmias and sudden cardiac death. Biventricular EF, QRS prolongation, older age at repair, prior palliative shunt, renal dysfunction, cardiothoracic ratio, $\mathrm{RV}$ hypertrophy, pulmonary regurgitation and right atrial (RA) area have been linked to adverse outcomes in patients with $\mathrm{rTOF}^{2-8}$ However, there remains a relative paucity of sensitive early biomarkers to detect adverse outcomes prior to onset of overt clinical signs or symptoms.

Neurohormonal activation has been recently reported in complex CHD, even among asymptomatic patients. ${ }^{9}{ }^{10}$ Brain-natriuretic peptide (BNP) and its precursor, $\mathrm{N}$-terminal pro-B-type natriuretic peptide (NT-proBNP) in particular, have emerged as potential peripheral biomarkers in acquired left heart failure, as their diagnostic and prognostic value is firmly established. ${ }^{11}{ }^{12}$ BNP in these patients is associated with functional capacity, LV dysfunction and mortality. By contrast, the role of neurohormonal activation in CHD remains illdefined, with limited longitudinal data.

A systematic review of 20 publications encompassing 770 patients with rTOF verified significantly elevated BNP levels in patients with rTOF compared with controls, which in turn correlated to the New York Heart Association (NYHA) class. ${ }^{10}$ The relationship between BNP with RV volumes and function was overall inconsistent, with non-significant correlations in some studies, but also evidence of positive correlations between BNP, $\mathrm{RV}$ volumes and the severity of pulmonary regurgitation in others. ${ }^{10}{ }^{13}$ We aimed to investigate the prognostic value of BNP in patients with rTOF in a prospective longitudinal study.

\section{PATIENTS AND METHODS}

Detailed clinical and neurohormonal assessment of adult patients with rTOF was prospectively undertaken between September 2000 and March 2004. Patients aged $\geq 16$ years attending tertiary adult CHD outpatient care at the Royal Brompton Hospital were recruited at the time of referral for Cardiovascular Magnetic Resonance (CMR), with follow-up for clinical events subsequently. All participants gave study-specific written informed consent after local ethics committee approval. Patients with clinically decompensated cardiac disease, evidence of active infection or malignancy were excluded.

CMR was acquired to quantify ventricular volumes, mass and function indexed to body surface area, in addition to pulmonary regurgitant fraction. CMR-derived measurements were analysed (SBN) blinded to venous sampling data. In accordance with 
the study protocol, patients had same day standard 12-lead ECG recorded (Hewlett Packard PageWriteXLi). Posteroanterior chest radiographs were also performed (Siemens Digiscan), allowing for cardiothoracic ratio calculation. Clinical data at baseline were recorded, including heart rate and blood pressure. Cardiopulmonary treadmill exercise testing data using the modified Bruce protocol was included in the analysis when performed within 6 months of the study recruitment date.

Peripheral blood samples were obtained for neurohormonal quantification from all patients in the morning as previously published. ${ }^{9}$ Atrial natriuretic peptide (ANP), BNP and active renin were quantified with immunoradiometric assays (BNP $1 \mathrm{pmol} /$ $\mathrm{L}=3.472 \mathrm{pg} / \mathrm{mL}$ ). Serum aldosterone was determined by radioimmunoassay (EuroDPC) and endothelin-1 (ET-1) was measured by ELISA (Bachem). Full blood count, renal function and liver function were assayed using routine laboratory methods.

Age-matched and gender-matched healthy controls were also recruited. Control subjects had their height, weight, heart rate and blood pressure recorded at recruitment in addition to peripheral venous blood sampling in accordance with the study protocol described.

The primary endpoint was all-cause mortality. A secondary endpoint of documented sustained arrhythmia (atrial or ventricular) was also analysed. Follow-up data was obtained from hospital medical records. Survival status was comprehensively assessed through the National Health Service electronic system linked to the national database of mortality data at the Office for National Statistics (UK).

\section{Statistical analysis}

Results for each variable were tested for normality with the Kolmogorov Smirnov method, with log transformation performed for non-parametric data for statistical analysis. Results are reported as mean $\pm S D$ or median (IQR), with groups assessed by unpaired Student $t$ test or Wilcoxon test as appropriate. Correlation analyses were assessed with Pearson's and Spearman correlation tests. Possible relationships between demographic, clinical and neurohormonal variables were ascertained by univariate Cox proportional hazard analysis. The low event rate did not permit multivariate analysis. HRs and twosided 95\% CIs are reported. Receiver operating curve (ROC) analyses were performed to assess the prognostic value of natriuretic peptide axis. The results were illustrated with KaplanMeier curves. Cut-off values were identified on survival-ROC method implemented in survival-ROC R-package using KaplanMeier estimate and the median follow-up time as timepoint of interest. Statistical analysis was performed with SPSS (IBM Statistics V.22) and R V.2.12.1. A two-sided p value $<0.05$ was considered significant.

\section{RESULTS}

\section{Baseline characteristics}

Ninety patients with rTOF were prospectively included (mean age $32.7 \pm 11.3$ years, 58 men). Fifteen age-matched and gendermatched healthy controls were also recruited for comparison. Baseline characteristics of both groups are presented in table 1. Forty-two patients had previous palliative shunts and median age at repair was $6(0.25-48.0)$ years. Transannular patching was required at surgical repair in $50 \%$ of patients. Twelve patients (13\%) had undergone previous surgical pulmonary valve replacement. Only a small proportion of patients with rTOF were on cardiac medications.

Patients with rTOF had significantly higher baseline heart rates when compared with controls (rTOF $84 \pm 14 \mathrm{bpm}$ vs controls $70 \pm 8 \mathrm{bpm}, \mathrm{p}<0.05)$, with no other significant difference in baseline characteristics.

Repaired TOF patients were, on average, normotensive, with broad QRS complexes of right bundle branch block pattern (mean QRS duration $147 \pm 28 \mathrm{~ms}$ ) and cardiomegaly (mean cardiothoracic ratio $0.55 \pm 0.06)$. Sixty-five $(72 \%)$ participants completed cardiopulmonary exercise testing with respiratory exchange ratio $>1$, thus their data were included in the analysis. Study participants had mean pulmonary regurgitation fraction $29.9 \pm 14.8 \%$. Sixty-four (71\%) study participants were asymptomatic in NYHA functional class I at the time of inclusion.

\section{Neurohormonal dysregulation in rTOF and cross-sectional relationships}

Neurohormonal activation was evident in patients with rTOF as compared with controls, outlined in table 1 and elevated levels of ANP, BNP, ET-1 and renin were found.

Seventy-one patients with rTOF (79\%) had abnormal BNP concentrations (ie, $\geq 5.3 \mathrm{pmol} / \mathrm{L}$ or $18.3 \mathrm{pg} / \mathrm{mL}$ ). ${ }^{14}$ Seventy per cent of asymptomatic patients had abnormal BNP levels. Symptomatic patients (NYHA functional class $\geq$ II) had higher ANP, BNP, renin and aldosterone levels (table 2). Symptoms also corresponded to recognised adverse clinical markers such as age, sodium, diminished peak oxygen consumption on cardiopulmonary exercise testing, higher cardiothoracic ratio and lower biventricular EF on CMR (with $\mathrm{p}<0.05$ ). Symptomatic patients appeared to paradoxically have lower pulmonary regurgitant fraction and QRS duration. The reason may be that we included patients who had undergone what in contemporary practice might be considered delayed pulmonary valve replacement $(13 \%)$ based predominantly on symptom onset. These patients will have had relief of pulmonary regurgitation and reduction in QRS duration but potentially incomplete symptom relief. While BNP levels were higher in women than in men in line with recognised gender differences (median BNP in women $10.5(7.6-22.8)$ vs $8.1(5.2-12.2) \mathrm{pmol} / \mathrm{L}$ in men), this was not statistically significant $(\mathrm{p}=0.42)$.

Significantly higher levels of ANP, BNP and ET-1 were evident in patients who had undergone previous palliation prior to repair, compared with patients who underwent primary repair $(\mathrm{p}<0.05)$. Elevated neurohormones were associated with older age at repair (notably ANP); Ln ANP-age at surgery $\mathrm{r}=0.55, \mathrm{p}<0.01$, Ln BNP-age at surgery $\mathrm{r}=0.25, \mathrm{p}=0.02$, Ln ET-1-age at surgery $r=0.33, p=0.01$.

Close relationships between the natriuretic peptides $(r=0.65)$ and renin/aldosterone $(\mathrm{r}=0.53)$ were evident (table 3$)$. Furthermore, there were significant correlations between ET-1 and natriuretic peptides (Ln BNP/Ln ET-1, r=0.83; Ln ANP/Ln ET-1, $r=0.39)$; in addition to ANP and aldosterone $(r=0.29)$, although weak. The natriuretic peptide axis (ANP/BNP) exhibited significant correlations with cardiothoracic ratio and indexed RA area (figure 1). Although correlations between BNP with RV end-diastolic volume and RVEF would not be surprising, this was not the case. However, symptomatic patients had higher LV end-diastolic volume and lower LVEF compared with the asymptomatic patients $(\mathrm{p}<0.05)$. There were no other significant associations.

\section{Outcomes}

Comprehensive follow-up data was available for 83 patients spanning a median period of 10 years (IQR $0.71-12.4$ years). Participants lost to follow-up were due to emigration $(n=3)$ and repeat defaulting from clinical attendance $(n=4)$. 
Table 1 Baseline demographics of patients with repaired tetralogy of Fallot (rTOF) vs controls (values represent mean \pm SD or median (IQR) as appropriate)

\begin{tabular}{|c|c|c|c|}
\hline & rTOF* $^{*}(n=90)$ & Controls $(n=15)$ & p Value \\
\hline Age (years) & $32.7 \pm 11.27$ & $30.3 \pm 4.99$ & 0.82 \\
\hline Gender (male/female) & $53 / 32$ & $7 / 8$ & \\
\hline Body Mass Index $\left(\mathrm{kg} / \mathrm{m}^{2}\right)$ & $24.4 \pm 4.94$ & $24.4 \pm 2.76$ & 0.92 \\
\hline Heart rate (bpm) & $84 \pm 15$ & $70 \pm 8$ & $<0.05$ \\
\hline Systolic blood pressure (mm Hg) & $116.6 \pm 15.2$ & $113.2 \pm 14.5$ & 0.45 \\
\hline Diastolic blood pressure $(\mathrm{mm} \mathrm{Hg})$ & $72.5 \pm 11.4$ & $73.5 \pm 8.5$ & 0.76 \\
\hline ANP (pmol/L) & $6.9(4.0-9.9)$ & $3.3(1.0-4.0)$ & $<0.05$ \\
\hline BNP (pmol/L) & $8.9(5.9-14.6)$ & $5.4(2.2-7.5)$ & $<0.05$ \\
\hline Endothelin-1 (pmol/L) & $1.14(0.94-1.48)$ & $0.75(0.44-0.93)$ & $<0.05$ \\
\hline Renin (pmol/L) & $55.0(45.5-66.5)$ & $18.6(12.0-22.7)$ & $<0.05$ \\
\hline Aldosterone (nmol/L) & $357(295-450)$ & $320(250-402)$ & 0.10 \\
\hline Haemoglobin (g/dL) & $14.8 \pm 1.64$ & $14.2 \pm 1.14$ & 0.21 \\
\hline Sodium (mmol/L) & $138.3 \pm 2.16$ & $137.4 \pm 1.84$ & 0.15 \\
\hline Creatinine (micmol/L) & $78.8 \pm 19.4$ & $76.8 \pm 9.6$ & 0.69 \\
\hline CRP (mg/L) & $7.51 \pm 3.57$ & $6.13 \pm 1.96$ & 0.16 \\
\hline QRS duration (ms) & $147 \pm 28.3$ & & \\
\hline Cardiothoracic ratio (\%) & $55 \pm 6$ & & \\
\hline \multicolumn{4}{|l|}{ Cardiopulmonary exercise testing ( $n=65$ with $R E R>1$ ) } \\
\hline Peak VO $2(\mathrm{~mL} / \mathrm{kg} / \mathrm{min})$ & $27.0 \pm 8.4$ & & \\
\hline Predicted peak $\mathrm{VO}_{2}(\%)$ & $79.4 \pm 18.1$ & & \\
\hline $\mathrm{VE} / \mathrm{VCO}_{2}(\mathrm{~mL} / \mathrm{kg} / \mathrm{min})$ & $29.5 \pm 7.4$ & & \\
\hline Anaerobic threshold (mL/kg/min) & $16.9 \pm 5.2$ & & \\
\hline \multicolumn{4}{|l|}{ Symptoms, n (\%) } \\
\hline NYHA Class I & $64(71)$ & & \\
\hline NYHA Class II & $20(22)$ & & \\
\hline NYHA Classes III and IV & $6(7)$ & & \\
\hline Age at primary repair (years) & 6.0 (IQR 0.25-48.0) & & \\
\hline \multicolumn{4}{|l|}{ Surgical history, n (\%) } \\
\hline Previous palliative procedure & $42(47)$ & & \\
\hline Blalock-Taussig shunt & $23(26)$ & & \\
\hline Waterston shunt & $13(14)$ & & \\
\hline Brock procedure & $6(7)$ & & \\
\hline \multicolumn{4}{|l|}{ Nature of repair, n (\%) } \\
\hline Transannular patch & $45(50)$ & & \\
\hline RVOT patch & $23(26)$ & & \\
\hline Conduit insertion & $11(12)$ & & \\
\hline Surgical history not available & $11(12)$ & & \\
\hline Redo surgery for pulmonary valve replacement & $12(13)$ & & \\
\hline \multicolumn{4}{|l|}{ Medications, n (\%) } \\
\hline Angiotensin-converting enzyme inhibitors/ angiotensin receptor blockers & $5(6)$ & & \\
\hline$\beta$ blockers & $6(7)$ & & \\
\hline Digoxin & $3(4)$ & & \\
\hline Diuretics & $9(11)$ & & \\
\hline Antiarrhythmics (Amiodarone, Sotalol) & $12(14)$ & & \\
\hline
\end{tabular}

Statistically significant $\mathrm{p}$ values are highlighted in bold.

${ }^{*}$ Nine $(10 \%)$ patients have pulmonary atresia subtype, 1 patient with absent pulmonary valve and 1 double outlet RV.

ANP, atrial natriuretic peptide; BNP, brain natriuretic peptide; VE/ $\mathrm{VCO}_{2}$, ventilatory efficiency; $\mathrm{VO}_{2}$, peak oxygen uptake; RVOT, RV outflow tract; NYHA, New York Heart Association;

CRP, C-reactive protein; RER, respiratory exchange ratio.

Primary endpoint: mortality

There were 8 deaths, with median age at death being 39.3 years (range 29.9-65.8). The causes of death included sudden cardiac death $(n=4)$, perioperative complications at the time of pulmonary valve replacement with acute RV failure $(n=2)$, and respiratory tract infection with sepsis $(n=1)$. The cause of death for a single patient (aged 30 years at death) could not be established, as local documentation is no longer available. Of the patients who died of sudden cardiac death, 2 patients experienced a presumed fatal arrhythmia within an hour of symptom onset, while a third patient experienced recurrent and eventually fatal arrhythmia, while in a persistent vegetative state, having sustained preceding aborted sudden death with ventricular fibrillation during pregnancy. The fourth patient had concomitant aortic stenosis.

When comparing the deceased patients with the rest of the study cohort, there was a trend towards higher BNP in deceased patients $(15.5(6.3-38.8) \mathrm{pmol} / \mathrm{L})$ compared to survivors $(8.9$ (5.9-14.1) $\mathrm{pmol} / \mathrm{L}), \mathrm{p}=0.53$. The deceased patients had significantly larger cardiothoracic ratios, higher RV volumes and 
Table 2 Neurohormonal activation and symptoms in tetralogy of Fallot patients

\begin{tabular}{|c|c|c|c|c|}
\hline & Total $(n=90)$ & Asymptomatic $(n=64)$ & Symptomatic $(n=26)$ & $p$ Value \\
\hline Age at study, years & $32.7 \pm 11.3$ & $31.0 \pm 10.6$ & $36.8 \pm 11.9$ & 0.03 \\
\hline \multicolumn{5}{|l|}{ Neurohormones } \\
\hline ANP (pmol/L) & $6.9(4.0-9.9)$ & $5.3(3.3-8.5)$ & $8.5(6.1-21.0)$ & $<0.05$ \\
\hline $\mathrm{BNP}(\mathrm{pmol} / \mathrm{L})$ & $8.9(5.9-14.6)$ & $8.9(5.8-12.7)$ & $11.4(6.2-33.7)$ & 0.04 \\
\hline Endothelin-1 (pmol/L) & $1.14(0.94-1.48)$ & $1.10(0.92-1.37)$ & $1.24(1.00-2.10)$ & 0.16 \\
\hline Renin (pmol/L) & $55.0(45.5-66.5)$ & $54.5(45.0-64.8)$ & $56.0(50.0-78.5)$ & 0.02 \\
\hline Aldosterone (nmol/L) & $357(295-450)$ & $346(295-400)$ & $485(355-787)$ & $<0.05$ \\
\hline \multicolumn{5}{|l|}{ Biochemical parameters } \\
\hline Haemoglobin (g/dL) & $14.8 \pm 1.64$ & $14.9 \pm 1.33$ & $14.6 \pm 2.25$ & 0.38 \\
\hline Sodium (mmol/L) & $138.3 \pm 2.16$ & $138.6 \pm 1.98$ & $137.4 \pm 2.33$ & 0.01 \\
\hline Creatinine (micmol/L) & $78.8 \pm 19.4$ & $77.5 \pm 14.6$ & $82.0 \pm 28.1$ & 0.32 \\
\hline Urate (micmol/L) & $325.6 \pm 79.0$ & $330.9 \pm 77.0$ & $313.0 \pm 83.6$ & 0.34 \\
\hline $\mathrm{CRP}(\mathrm{mg} / \mathrm{L})$ & $7.51 \pm 3.57$ & $7.39 \pm 2.39$ & $7.74 \pm 5.19$ & 0.78 \\
\hline Cardiopulmonary exercise testing (RER $>1$ ) & & $(n=52)$ & $(n=13)$ & \\
\hline Peak $\mathrm{VO}_{2}(\mathrm{~mL} / \mathrm{kg} / \mathrm{min})$ & $27.0 \pm 8.4$ & $28.1 \pm 8.7$ & $22.3 \pm 5.1$ & $<0.05$ \\
\hline Predicted peak $\mathrm{VO}_{2}(\%)$ & $79.4 \pm 18.1$ & $82.7 \pm 17.6$ & $65.7 \pm 13.5$ & $<0.05$ \\
\hline $\mathrm{VE} / \mathrm{VCO}_{2}$ & $29.5 \pm 7.4$ & $29.0 \pm 7.4$ & $31.2 \pm 7.1$ & 0.34 \\
\hline Anaerobic threshold (mL/kg/min) & $16.9 \pm 5.2$ & $17.5 \pm 7.3$ & $14.6 \pm 4.5$ & 0.12 \\
\hline QRS duration (ms) & $147 \pm 28.3$ & $145 \pm 26.3$ & $131 \pm 21.5$ & 0.04 \\
\hline Cardiothoracic ratio & $0.55 \pm 0.06$ & $0.54 \pm 0.05$ & $0.60 \pm 0.07$ & $<0.05$ \\
\hline \multicolumn{5}{|l|}{ CMR parameters } \\
\hline RVEDV indexed $\left(\mathrm{mL} / \mathrm{m}^{2}\right)$ & $130.1 \pm 44.7$ & $125.1 \pm 34.9$ & $143.6 \pm 63.1$ & 0.40 \\
\hline RVESV indexed $\left(\mathrm{mL} / \mathrm{m}^{2}\right)$ & $64.3 \pm 36.7$ & $58.3 \pm 24.5$ & $80.5 \pm 55.7$ & 0.13 \\
\hline RVSV indexed $\left(\mathrm{mL} / \mathrm{m}^{2}\right)$ & $65.6 \pm 18.6$ & $66.5 \pm 18.9$ & $63.2 \pm 17.9$ & 0.48 \\
\hline RVEF $(\%)$ & $52.3 \pm 11.3$ & $53.9 \pm 10.5$ & $48.1 \pm 17.9$ & 0.03 \\
\hline $\mathrm{RV}$ indexed mass $\left(\mathrm{g} / \mathrm{m}^{2}\right)$ & $57.3 \pm 17.4$ & $56.5 \pm 14.0$ & $59.6 \pm 24.7$ & 0.58 \\
\hline LVEDV indexed $\left(\mathrm{mL} / \mathrm{m}^{2}\right)$ & $72.5 \pm 18.8$ & $69.4 \pm 15.4$ & $80.7 \pm 24.4$ & 0.02 \\
\hline LVESV indexed $\left(\mathrm{mL} / \mathrm{m}^{2}\right)$ & $27.1 \pm 10.4$ & $24.7 \pm 8.2$ & $33.3 \pm 13.2$ & $<0.05$ \\
\hline LVSV indexed $\left(\mathrm{mL} / \mathrm{m}^{2}\right)$ & $45.5 \pm 11.7$ & $44.7 \pm 10.4$ & $47.9 \pm 14.7$ & 0.34 \\
\hline $\operatorname{LVEF}(\%)$ & $63.4 \pm 7.9$ & $64.6 \pm 7.7$ & $60.3 \pm 8.0$ & 0.03 \\
\hline $\mathrm{LV}$ indexed mass $\left(\mathrm{g} / \mathrm{m}^{2}\right)$ & $63.7 \pm 18.7$ & $63.0 \pm 13.7$ & $65.5 \pm 28.4$ & 0.69 \\
\hline$\geq$ Moderate pulmonary stenosis* ${ }^{*} \mathrm{n}$ & 8 & 4 & 4 & 0.17 \\
\hline$\geq$ Moderate pulmonary regurgitation, $\mathrm{n}$ & 78 & 56 & 22 & 0.72 \\
\hline$\geq$ Moderate pulmonary regurgitation or stenosis, $n$ & 80 & 58 & 22 & 0.41 \\
\hline Pulmonary regurgitant fraction (\%) & $29.9 \pm 14.8$ & $33.0 \pm 14.2$ & $21.6 \pm 13.3$ & $<0.05$ \\
\hline
\end{tabular}

*Moderate pulmonary stenosis=peak velocity $\geq 3 \mathrm{~m} / \mathrm{s}$.

ANP, atrial natriuretic peptide; BNP, brain natriuretic peptide; CMR, cardiovascular magnetic resonance; LVEDV, LV end-diastolic volume; LVESV, LV end-systolic volume; LVSV, LV stroke volume; RVEDV, RV end-diastolic volume; RVESV, RV end-systolic volume; RVSV, RV stroke volume; CRP, C-reactive protein; $\mathrm{VE} / \mathrm{VCO}_{2}$, ventilatory efficiency; VO${ }_{2}$, peak oxygen uptake; RER, respiratory exchange ratio.

Table 3 Correlation coefficients of neurohormones in patients with repaired tetralogy of Fallot

\begin{tabular}{|c|c|c|c|c|c|}
\hline & $\begin{array}{l}\text { Ln } \\
\text { ANP }\end{array}$ & Ln BNP & Ln ET-1 & Ln Renin & $\begin{array}{l}\text { Ln } \\
\text { Aldosterone }\end{array}$ \\
\hline Ln ANP & & $\begin{array}{l}r=0.65 \\
p<0.01\end{array}$ & $\begin{array}{l}r=0.39 \\
p<0.01\end{array}$ & $\begin{array}{l}r=0.04 \\
p=0.72\end{array}$ & $\begin{array}{l}r=0.29 \\
p=0.01\end{array}$ \\
\hline Ln BNP & & & $\begin{array}{l}r=0.83 \\
p<0.01\end{array}$ & $\begin{array}{l}r=-0.03 \\
p=0.81\end{array}$ & $\begin{array}{l}r=0.18 \\
p=0.11\end{array}$ \\
\hline Ln ET-1 & & & & $\begin{array}{l}r=0.14 \\
p=0.33\end{array}$ & $\begin{array}{l}r=0.23 \\
p=0.10\end{array}$ \\
\hline Ln renin & & & & & $\begin{array}{l}r=0.53 \\
p<0.01\end{array}$ \\
\hline $\begin{array}{l}\text { Ln } \\
\text { aldosterone }\end{array}$ & & & & & \\
\hline
\end{tabular}

ANP, atrial natriuretic peptide; BNP, brain natriuretic peptide; ET-1, endothelin-1. bi-atrial dilatation when compared with the rest of the study cohort (table 4). All deceased patients had residual haemodynamic lesions at baseline with 7 patients having at least moderate pulmonary regurgitation and the last patient having aortic stenosis.

BNP was significantly related to all-cause mortality on univariate Cox proportional hazard analysis (HR 1.16 per $10 \mathrm{pmol} / \mathrm{L}$, $95 \%$ CI 1.05 to $1.29, \mathrm{p}<0.01$ ); as illustrated in table $5 \mathrm{~A}$ and figure $2 \mathrm{~A}$. Other predictors of mortality include recognised adverse markers of clinical outcome, including cardiothoracic ratio, creatinine and indexed RA area. Time-dependent ROC characteristics analysis supported the predictive value of BNP at 5 years of follow-up with BNP $\geq 15 \mathrm{pmol} / \mathrm{L}$ related to increased mortality (HR $5.40,95 \%$ CI 1.29 to $22.60, \mathrm{p}<0.01$, figure $2 \mathrm{~B}$ ). The absolute mortality at 5 -year follow-up was $19 \%$ (95\% CI 0.4 to 34.2 ) in patients with $\mathrm{BNP} \geq 15 \mathrm{pmol} / \mathrm{L}$, by contrast with $3 \%$ (95\% CI 0.0 to 7.5 ) in patients with $\mathrm{BNP} \leq 15 \mathrm{pmol} / \mathrm{L}$. 

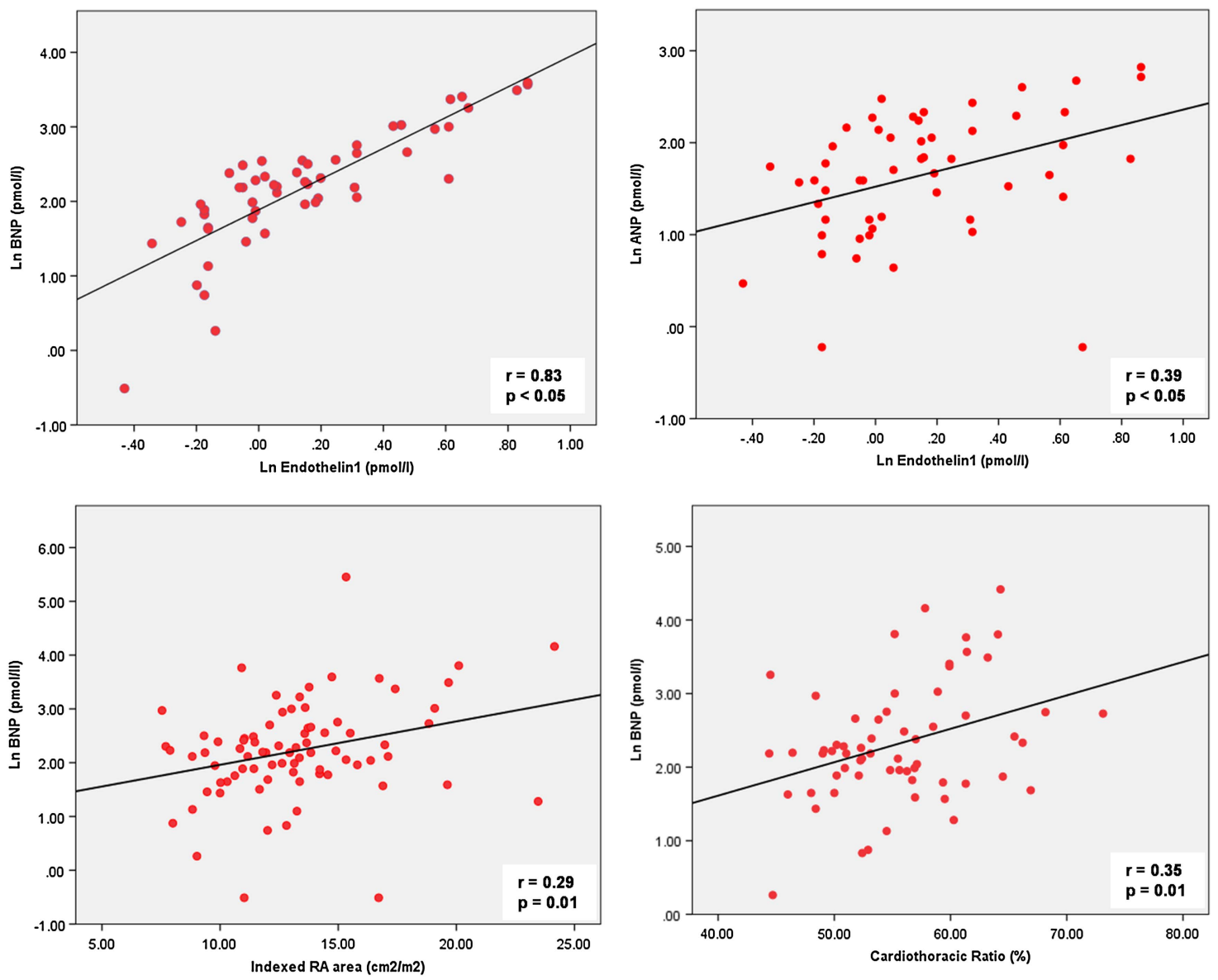

Figure 1 Neurohormonal and clinical variable interactions in repaired tetralogy of Fallot. ANP, atrial natriuretic peptide; BNP, brain natriuretic peptide; RA, right atrial.

Volumetric and functional parameters on CMR and indices of cardiopulmonary exercise capacity were not associated with death.

\section{Secondary endpoint: sustained atrial or ventricular} tachy-arrhythmia

There were 28 events for the secondary outcome of documented sustained arrhythmia (atrial and/or ventricular). Nineteen patients had 20 atrial arrhythmias (atrial flutter $n=11$, atrial fibrillation $\mathrm{n}=6$, atrioventricular nodal re-entrant tachycardia $\mathrm{n}=3$ ), whereas 7 patients had 8 documented ventricular arrhythmias (ventricular tachycardia $n=6$, ventricular fibrillation $\mathrm{n}=2$ ). Ten patients required direct-current cardioversion for restoration of sinus rhythm.

Univariate neurohormonal predictors of sustained arrhythmia on Cox proportional hazards analysis include ANP, BNP and ET-1; together with other recognised adverse markers including patient age, QRS duration, exercise capacity, cardiothoracic ratio and indexed RA area (table 5B).

\section{DISCUSSION}

This study demonstrates elevated circulating levels of natriuretic peptides, ET-1 and renin in patients with rTOF. In particular, BNP was found to be predictive of mortality. Raised BNP levels were evident even in asymptomatic patients, with the extent of neurohormonal activation corresponding with functional class. Furthermore, there was interaction of ET-1 with the natriuretic peptide axis, which may provide new mechanistic insight.
The prognostic value of BNP

While we and others have demonstrated cross-sectional data of neurohormonal activation in rTOF, our current study suggests a prognostic value of BNP for patients with rTOF, with a BNP $\geq 15 \mathrm{pmol} / \mathrm{L}$ in our dataset predicting a fivefold increase in mortality. CHD, by definition, constitutes structural cardiac abnormality, negatively affects exercise capacity causing neurohormonal activation despite previous reparative surgery; and hence has been likened to chronic heart failure syndrome. ${ }^{15}$ As BNP is secreted by cardiomyocytes due to increased myocardial wall stress, ${ }^{16}$ it is consequently released in CHD in response to pressure or volume overload states. ${ }^{17} 18$

BNP is a sensitive marker of LV ventricular dysfunction, even in asymptomatic patients, and rises commensurate with the degree of LV dysfunction. ${ }^{4} 19$ The prognostic value of BNP in LV pathology is known, and has been shown to be a predictor of sudden death in chronic heart failure. ${ }^{20}$ The attributes of BNP in the left-sided chronic heart failure setting may therefore be appropriately extended to chronic RV pathology in rTOF, as ventricular myocyte stretch is recognised in rTOF ventricles most commonly subjected to chronic volume loading with pulmonary regurgitation. As sudden cardiac death in rTOF remains a poorly mitigated outcome, with a long-term estimated risk of $3.7 \%-6 \%,{ }^{6}{ }^{21}$ BNP measurements may have a role in the clinical risk assessment and decision-making process in primary prevention against sudden cardiac death.

There are limited data on the prognostic value of BNP in patients with rTOF. Our institution had demonstrated 
Table 4 Characteristics of deceased patients with repaired tetralogy of Fallot

\begin{tabular}{|c|c|c|c|c|}
\hline & Total $(n=83)$ & Alive $(n=75)$ & Deceased $(n=8)$ & $\mathrm{p}$ Value \\
\hline Age at study, years & $32.7 \pm 11.6$ & $32.2 \pm 11.3$ & $37.5 \pm 13.4$ & 0.22 \\
\hline \multicolumn{5}{|l|}{ Neurohormones } \\
\hline ANP (pmol/L) & $6.9(3.8-10.3)$ & $6.9(3.5-9.9)$ & $7.4(4.7-51.0)$ & 0.30 \\
\hline BNP (pmol/L) & $9.0(5.9-15.3)$ & $8.9(5.9-14.1)$ & $15.5(6.3-38.8)$ & 0.53 \\
\hline Endothelin-1 (pmol/L) & $1.06(0.93-1.37)$ & $1.10(0.95-1.37)$ & $0.78(0.65-0.78)$ & 0.20 \\
\hline Renin (pmol/L) & $55.0(45.0-67.0)$ & $55.0(45.0-68.0)$ & $52.0(45.3-64.0)$ & 0.40 \\
\hline Aldosterone (nmol/L) & $357.0(295.0-451.3)$ & $355.0(295.0-415.3)$ & $576.0(276.3-788.0)$ & 0.18 \\
\hline \multicolumn{5}{|l|}{ Biochemical parameters } \\
\hline Haemoglobin $(\mathrm{g} / \mathrm{dL})$ & $14.7 \pm 1.66$ & $14.8 \pm 1.63$ & $14.4 \pm 2.05$ & 0.38 \\
\hline Sodium (mmol/L) & $138.3 \pm 2.11$ & $138.3 \pm 2.15$ & $138.5 \pm 1.77$ & 0.77 \\
\hline Creatinine (micmol/L) & $77.8 \pm 19.4$ & $76.1 \pm 14.5$ & $94.4 \pm 43.5$ & 0.28 \\
\hline Urate (micmol/L) & $325.6 \pm 79.3$ & $326.6 \pm 81.5$ & $316.8 \pm 58.0$ & 0.74 \\
\hline CRP (mg/L) & $5.06 \pm 4.33$ & $7.84 \pm 3.72$ & $6.50 \pm 2.81$ & 0.40 \\
\hline Cardiopulmonary exercise testing $(R E R>1)$ & & $(n=58)$ & $(n=7)$ & \\
\hline Peak $\mathrm{VO}_{2}(\mathrm{~mL} / \mathrm{kg} / \mathrm{min})$ & $26.3 \pm 8.1$ & $26.6 \pm 8.1$ & $23.8 \pm 8.3$ & 0.35 \\
\hline Predicted peak $\mathrm{VO}_{2}(\%)$ & $77.1 \pm 19.2$ & $77.8 \pm 19.2$ & $71.0 \pm 18.8$ & 0.38 \\
\hline VE/VCO ${ }_{2}(\mathrm{~mL} / \mathrm{kg} / \mathrm{min})$ & $30.6 \pm 8.0$ & $30.4 \pm 7.9$ & $31.8 \pm 9.6$ & 0.67 \\
\hline Anaerobic threshold (mL/kg/min) & $15.9 \pm 4.8$ & $16.1 \pm 4.9$ & $14.2 \pm 3.3$ & 0.36 \\
\hline QRS duration (ms) & $145 \pm 28$ & $144 \pm 28$ & $148 \pm 31$ & 0.70 \\
\hline Cardiothoracic ratio & $0.56 \pm 0.06$ & $0.55 \pm 0.06$ & $0.65 \pm 0.07$ & $<0.05$ \\
\hline \multicolumn{5}{|l|}{ CMR parameters } \\
\hline RVEDV indexed $\left(\mathrm{mL} / \mathrm{m}^{2}\right)$ & $128.1 \pm 43.5$ & $124.5 \pm 37.6$ & $165.6 \pm 78.0$ & 0.22 \\
\hline RVESV indexed $\left(\mathrm{mL} / \mathrm{m}^{2}\right)$ & $62.3 \pm 34.7$ & $59.1 \pm 27.9$ & $95.6 \pm 70.8$ & 0.03 \\
\hline RVSV indexed $\left(\mathrm{mL} / \mathrm{m}^{2}\right)$ & $65.6 \pm 19.2$ & $65.1 \pm 19.3$ & $70.0 \pm 18.9$ & 0.52 \\
\hline $\operatorname{RVEF}(\%)$ & $52.7 \pm 10.7$ & $53.4 \pm 10.5$ & $46.1 \pm 11.6$ & 0.09 \\
\hline $\mathrm{RV}$ indexed mass $\left(\mathrm{g} / \mathrm{m}^{2}\right)$ & $56.7 \pm 17.2$ & $55.8 \pm 16.6$ & $65.3 \pm 22.4$ & 0.17 \\
\hline LVEDV indexed $\left(\mathrm{mL} / \mathrm{m}^{2}\right)$ & $72.5 \pm 19.4$ & $71.4 \pm 17.9$ & $84.0 \pm 30.2$ & 0.43 \\
\hline LVESV indexed $\left(\mathrm{mL} / \mathrm{m}^{2}\right)$ & $26.9 \pm 10.6$ & $45.6 \pm 11.5$ & $48.0 \pm 16.2$ & 0.17 \\
\hline LVSV indexed $\left(\mathrm{mL} / \mathrm{m}^{2}\right)$ & $45.8 \pm 11.9$ & $44.7 \pm 10.4$ & $47.9 \pm 14.7$ & 0.60 \\
\hline LVEF $(\%)$ & $63.5 \pm 8.0$ & $63.3 \pm 19.0$ & $70.7 \pm 21.6$ & 0.33 \\
\hline $\mathrm{LV}$ indexed mass $\left(\mathrm{g} / \mathrm{m}^{2}\right)$ & $63.9 \pm 19.2$ & $63.0 \pm 13.7$ & $65.5 \pm 28.4$ & 0.69 \\
\hline Indexed RA area $\left(\mathrm{cm}^{2} / \mathrm{m}^{2}\right)$ & $13.2 \pm 3.4$ & $12.9 \pm 3.2$ & $16.9 \pm 3.3$ & $<0.01$ \\
\hline Indexed LA area $\left(\mathrm{cm}^{2} / \mathrm{m}^{2}\right)$ & $9.4 \pm 2.4$ & $9.3 \pm 2.3$ & $12.0 \pm 2.8$ & 0.03 \\
\hline Pulmonary regurgitant fraction (\%) & $29.9 \pm 14.8$ & $33.5 \pm 11.4$ & $32.8 \pm 7.6$ & 0.90 \\
\hline
\end{tabular}

ANP, atrial natriuretic peptide; BNP, brain natriuretic peptide; CMR, cardiovascular magnetic resonance; LVEDV, LV end-diastolic volume; LVESV, LV end-systolic volume; LVSV, LV stroke volume; RVEDV, RV end-diastolic volume; RVESV, RV end-systolic volume; RVSV, RV stroke volume; RA, right atrial; LA, left atrial; CRP, C-reactive protein; RER, respiratory exchange ratio; $\mathrm{VE} / \mathrm{VCO}_{2}$, ventilatory efficiency; $\mathrm{VO}_{2}$, peak oxygen uptake.

previously that both ANP and BNP were strong predictors of mortality in a prospectively studied mixed cohort of 49 patients with symptomatic complex adult congenital heart disease, including 18 patients with rTOF. Fifty-seven per cent of that study cohort had systemic ventricular dysfunction. ${ }^{22}$ By comparison, our study shows that BNP remains of prognostic value within a single condition in a mostly asymptomatic cohort with generally preserved biventricular function. We, therefore, submit it may have applicability and clinical use in lifelong clinical management. Although BNP elevation observed in adult patients with rTOF were modest compared with patients with chronic heart failure, it did, however, provide prognostic information.

\section{Dysregulation of ET-1 in rTOF}

ET-1 was independently associated with the natriuretic peptide axis (Ln BNP/Ln ET-1, $\mathrm{r}=0.83$; Ln ANP/Ln ET-1, $\mathrm{r}=0.39$; $\mathrm{p}<0.05)$, and found to be elevated in patients with palliative shunts prior to surgical repair. ET-1 was isolated in 1988 as a potent vasoconstrictor and growth factor for myocardial fibroblast proliferation, thereby implicated in cardiac remodelling stimulating cardiac hypertrophy through fibrosis. ${ }^{23}$ Increased
ET-1 levels seen in chronic heart failure may be due to pulmonary congestion upregulating pulmonary endothelin-converting enzyme-1, which results in higher pulmonary production and secretion of ET-1 in a porcine model. ${ }^{24}$

Raised ET-1 levels are recognised in children with CHD, postulated to be related to combinations of increased pulmonary blood flow or pressure. The mechanism of elevated ET-1 in rTOF may, therefore, relate to the exposure of pulmonary vascular endothelium to increased shear forces with pulmonary regurgitation and large RV stroke volume, or residual pulmonary stenosis. ${ }^{25}$ This is further supported by higher ET-1 levels in patients with preceding palliative shunts to increase pulmonary blood flow with chronic LV volume loading in our study cohort. ET-1 is increased in patients with chronic heart failure with a similar relationship between ET-1 and the natriuretic peptide axis demonstrated, suggesting that neurohormonal activation may have pathophysiological links to circulating ET-1 levels. ${ }^{26}$ Raised ET-1 has prognostic implications in chronic heart failure, ${ }^{27}$ which may serve as a relevant peripheral biomarker if extended to rTOF, in which the pathophysiology involves progressive pulmonary regurgitation, cardiac remodelling and myocardial fibrosis. 
Table 5 Univariate predictors of outcome (A) mortality and (B) sustained arrhythmia

\begin{tabular}{llr}
\hline Variable & HR (95\% Cl) & p Value \\
\hline (A) Mortality & & \\
$\quad$ BNP, per 10 pmol/L & $1.16(1.05$ to 1.29$)$ & 0.01 \\
Cardiothoracic ratio, per $1 \%$ & $1.40(1.07$ to 1.83$)$ & 0.02 \\
Creatinine, per $10 \mathrm{micmol} / \mathrm{L}$ & $1.39(1.12$ to 1.73$)$ & $<0.01$ \\
RA indexed area, cm $/ \mathrm{m}^{2}$ & $1.23(1.01$ to 1.49$)$ & 0.04 \\
(B) Sustained arrhythmia & & \\
Age at study, years & $1.04(1.00$ to 1.07$)$ & 0.03 \\
Age at repair, years & $1.04(1.00$ to 1.08$)$ & 0.04 \\
QRS duration on ECG, per $10 \mathrm{~ms}$ & $1.20(1.02$ to 1.42$)$ & 0.03 \\
Peak VO ${ }_{2}, \mathrm{~mL} / \mathrm{kg} / \mathrm{min}$ & $0.93(0.86$ to 1.00$)$ & 0.04 \\
Cardiothoracic ratio, per $1 \%$ change & $1.24(1.12$ to 1.37$)$ & $<0.05$ \\
ANP, pmol/L & $1.77(1.21$ to 2.59$)$ & $<0.05$ \\
BNP, per 10 pmol/L & $2.06(1.32$ to 3.21$)$ & $<0.05$ \\
Endothelin-1, pmol/L & $8.18(1.29$ to 51.82$)$ & 0.03 \\
RVESVi, $\mathrm{mL} / \mathrm{m}^{2}$ & $2.46(1.14$ to 5.32$)$ & 0.02 \\
RA indexed area, cm $/ \mathrm{m}^{2}$ & $1.13(1.03$ to 1.24$)$ & 0.01 \\
\hline
\end{tabular}

ANP, atrial natriuretic peptide; BNP, brain natriuretic peptide; RVESV, RV end-systolic volume; $\mathrm{VO}_{2}$, peak oxygen uptake; $\mathrm{RA}$, right atrial.

\section{Applicability of neurohormone measurement to clinical practice}

The natriuretic peptide axis is associated with cardiothoracic ratio and indexed RA area $(\mathrm{p}<0.05)$, supporting mechanistic theories of RA size and myocyte stretch stimulating ANP and BNP release. ${ }^{9}{ }^{28}$ As the cardiothoracic ratio is dependent on the transverse diameter of the cardiac silhouette bordered by the right atrium and $\mathrm{LV}$ in atrioventricular-concordant hearts, the interdependence of cardiothoracic ratio and the natriuretic peptide axis is not surprising. These correlations between neurohormones and clinical variables are relevant in understanding the extent of neurohormonal activation in patients with rTOF.

Plasma BNP and NT-proBNP levels have variable correlations to exercise capacity, RV volumes and function in the literature with small series demonstrating relationships between BNP and RV volumes, dysfunction or pulmonary regurgitation. ${ }^{10}$ However, in keeping with our study, others have shown modest or no significant correlations of BNP to pulmonary regurgitation, exercise capacity or CMR-derived measures. ${ }^{29}$ These discrepancies may be due to differences in the selection, age group and sample size of patients studied. Furthermore, some patients with rTOF in contemporary practice undergo delayed pulmonary valve replacement, which restores pulmonary valve competency but with persistent RV dilatation that can elevate BNP values. Our data reflect 90 clinically stable adult patients, most of who did not meet current criteria based on CMR RV volumes for surgery, with preserved systolic function and only mildly impaired peak $\mathrm{VO}_{2}$.

BNP is currently measured routinely in the clinical management of patients with rTOF in numerous adult CHD centres, although its sensitivity and interpretation in the context of decision making for pulmonary valve replacement and risk stratification mandates further clarification. Neurohormonal modulation remains an important therapeutic target in view of early neurohormonal activation in this patient group, with limited evidencebase presently. ${ }^{30}$ Future randomised controlled studies with larger patient numbers, longer periods of observation and serial neurohormonal assays should be considered to assess this further. For the time being, addressing haemodynamic targets with surgical and/or catheter intervention in a timely fashion rather than neurohormonal antagonism remains the mainstay of therapy.

\section{Limitations}

This study was performed at a tertiary adult CHD centre, with study recruitment from patients referred for CMR. Selection bias towards patients with haemodynamically significant lesions may have potentially been introduced, as CMR may not have been routinely performed in all patients with rTOF under follow-up in this early era. Furthermore, patients with rTOF with implanted devices (pacemakers, cardiac resynchronisation devices or implantable cardioverter defibrillators) had to be excluded by necessity from the CMR-based recruitment. Nevertheless, our study reflects a large cohort of patients, many of them asymptomatic, and mostly stable who constitute the very population who need and benefit from risk assessment.

While BNP was predictive of all-cause mortality in the study cohort, the low event rate prevented accounting for other known adverse markers of clinical outcome and confounding factors. As such, our findings may have limited generalisability. Therefore, investigations in other large contemporary adult rTOF cohorts are justified. Further, prospective work is necessary to determine the relative, incremental value of serial BNP measurements in rTOF and examine its potential response to therapy.

\section{CONCLUSION}

In our single-centre prospective study with long-term follow-up data, neurohormonal activation was present in patients with rTOF and correlated with symptoms. We observed, for the first
Figure 2 (A) Kaplan-Meier survival curve (dotted lines represent $95 \% \mathrm{Cls}$ ). (B) Receiver operating curve survival analysis for brain natriuretic peptide (BNP) (AUC, area under the curve).

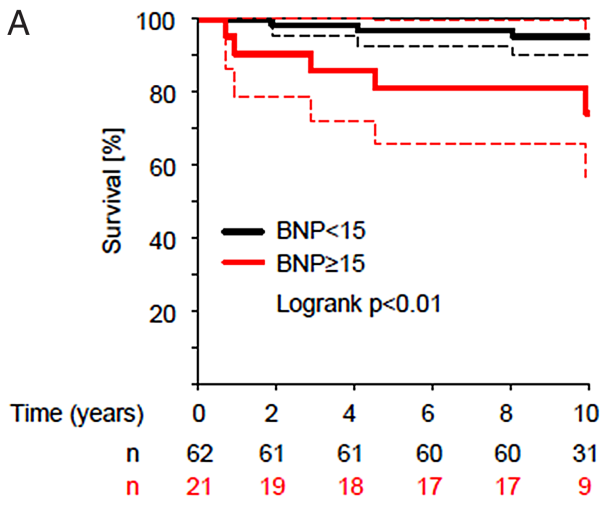


time, potentially pertinent mechanistic interactions between the natriuretic peptide axis and ET- 1 in these patients. The early pathological neurohormone profile remains a potential therapeutic target that merits further exploration. BNP may predict mortality and sustained arrhythmia late after rTOF, and merits serial assessment to define its clinical use for the lifelong care of these patients.

\section{Key messages}

\section{What is already known on this subject?}

Cross-sectional studies have showed there is neurohormonal activation including brain natriuretic peptide (BNP) in adults with repaired tetralogy of Fallot (rTOF). However, the use of BNP and its longitudinal predictive value of adverse outcomes in rTOF are unknown.

\section{What might this study add?}

This large, prospective study demonstrates neurohormonal activation even in asymptomatic patients with rTOF, with a prognostic role of BNP in predicting mortality and arrhythmias. A BNP level $\geq 15 \mathrm{pmol} / \mathrm{L}$ is associated with a fivefold increased risk of death. Mechanistic interactions of neurohormones, in particular of BNP and endothelin-1, were evident.

\section{How might this impact on clinical practice?}

Elevated BNP levels in asymptomatic patients with rTOF may serve as an early clinical marker of underlying cardiac dysfunction before the often late onset of symptoms. The relationship between BNP, mortality and arrhythmias could be exploited as a potential therapeutic target for symptomatic and prognostic benefit. The measurement of BNP in this patient cohort has clinical use for risk stratification, and could be integrated into the armamentarium of clinical biomarkers during long-term care.

Contributors ELH: data collation, analysis and interpretation, manuscript drafting; APB: design and conduct of study; AK: data analysis; PAD: acquisition of CMR data; SVB-N: acquisition and blinded analysis of CMR data, interpretation and manuscript drafting. All authors contributed to critical and intellectual revision of manuscript. All authors have given approval of manuscript for publication.

Funding ELH is supported by a Clinical Research Training Fellowship from the British Heart Foundation (FS/13/76/30477). SVB-N is supported by an Intermediate Clinical Research Fellowship from the British Heart Foundation (FS/11/38/28864). This project was supported by the NIHR Cardiovascular Biomedical Research Unit of the Royal Brompton and Harefield NHS Foundation Trust and Imperial College London. This report is independent research by the National Institute for Health Research Biomedical Research Unit Funding Scheme. The views expressed in this publication are those of the author(s) and not necessarily those of the NHS, the National Institute for Health Research or the Department of Health.

Competing interests DJP is a consultant to Siemens and a shareholder and director of Cardiovascular Imaging Solutions. MAG has served on the advisory board of Actelion, Pfizer and GlaxoSmithKline and has received unrestricted educational grants from Actelion and Pfizer, UK.

Ethics approval Brompton, Harefield and NHLI Research Ethics Committee. Provenance and peer review Not commissioned; externally peer reviewed.

\section{REFERENCES}

1 Baumgartner $\mathrm{H}$, Bonhoeffer $\mathrm{P}$, De Groot NM, et al. ESC guidelines for the management of grown-up congenital heart disease (new version 2010). Eur Heart 2010;31:2915-57.

2 Orwat S, Diller GP. Risk stratification in adults with repaired tetralogy of fallot: The long journey from clinical parameters and surface ecg to in-depth assessment of myocardial mechanics, volume and pressure loading. Heart 2014;100:185-7.
3 Ghai $A$, Silversides $C$, Harris $L$, et al. Left ventricular dysfunction is a risk factor for sudden cardiac death in adults late after repair of tetralogy of fallot. J Am Coll Cardiol 2002;40:1675-80

4 Diller GP, Kempny A, Liodakis E, et al. Left ventricular longitudinal function predicts life-threatening ventricular arrhythmia and death in adults with repaired tetralogy of fallot. Circulation 2012;125:2440-6.

5 Valente AM, Gauvreau K, Assenza GE, et al. Contemporary predictors of death and sustained ventricular tachycardia in patients with repaired tetralogy of fallot enrolled in the indicator cohort. Heart 2014;100:247-53.

6 Gatzoulis MA, Balaji S, Webber SA, et al. Risk factors for arrhythmia and sudden cardiac death late after repair of tetralogy of fallot: A multicentre study. Lancet 2000;356:975-81.

7 Bonello B, Kempny A, Uebing A, et al. Right atrial area and right ventricular outflow tract akinetic length predict sustained tachyarrhythmia in repaired tetralogy of fallot. Int J Cardiol 2013;168:3280-6.

8 Katz NM, Blackstone EH, Kirklin JW, et al. Late survival and symptoms after repair of tetralogy of fallot. Circulation 1982;65:403-10.

9 Bolger AP, Sharma R, Li W, et al. Neurohormonal activation and the chronic heart failure syndrome in adults with congenital heart disease. Circulation 2002; 106:92-9.

10 Eindhoven JA, van den Bosch $A E$, Jansen PR, et al. The usefulness of brain natriuretic peptide in complex congenital heart disease: A systematic review. J Am Coll Cardiol 2012;60:2140-9

11 Nagaya N, Nishikimi T, Okano Y, et al. Plasma brain natriuretic peptide levels increase in proportion to the extent of right ventricular dysfunction in pulmonary hypertension. J Am Coll Cardiol 1998;31:202-8.

12 de Lemos JA, Morrow DA, Bentley JH, et al. The prognostic value of b-type natriuretic peptide in patients with acute coronary syndromes. $N$ Engl J Med 2001;345:1014-21.

13 Cheung EW, Lam WW, Chiu CS, et al. Plasma brain natriuretic peptide levels, right ventricular volume overload and exercise capacity in adolescents after surgical repair of tetralogy of fallot. Int J Cardiol 2007;121:155-62.

14 Shionoria BNP immunoradiometryc assay kit product insert. http://www.radmed. com.tr/usr_img/urunler/bnp2.pdf (accessed Aug 2014)

15 Bolger AP, Coats AJ, Gatzoulis MA. Congenital heart disease: the original heart failure syndrome. Eur Heart J 2003;24:970-6.

16 Mukoyama M, Nakao K, Hosoda K, et al. Brain natriuretic peptide as a novel cardiac hormone in humans. Evidence for an exquisite dual natriuretic peptide system, atrial natriuretic peptide and brain natriuretic peptide. J Clin Invest 1991;87:1402-12.

17 Tulevski II, Groenink M, van Der Wall EE, et al. Increased brain and atrial natriuretic peptides in patients with chronic right ventricular pressure overload: correlation between plasma neurohormones and right ventricular dysfunction. Heart 2001:86:27-30.

18 Hopkins WE, Chen Z, Fukagawa NK, et al. Increased atrial and brain natriuretic peptides in adults with cyanotic congenital heart disease: Enhanced understanding of the relationship between hypoxia and natriuretic peptide secretion. Circulation 2004;109:2872-7.

19 McDonagh TA, Robb SD, Murdoch DR, et al. Biochemical detection of left-ventricular systolic dysfunction. Lancet 1998;351:9-13.

20 Berger R, Huelsman M, Strecker K, et al. B-type natriuretic peptide predicts sudden death in patients with chronic heart failure. Circulation 2002;105:2392-7.

21 Harrild DM, Berul $\mathrm{Cl}$, Cecchin $\mathrm{F}$, et al. Pulmonary valve replacement in tetralogy of fallot: Impact on survival and ventricular tachycardia. Circulation 2009;119:445-51.

22 Giannakoulas G, Dimopoulos K, Bolger AP, et al. Usefulness of natriuretic peptide levels to predict mortality in adults with congenital heart disease. Am J Cardiol 2010;105:869-73.

23 Yanagisawa $\mathrm{M}$, Kurihara $\mathrm{H}$, Kimura $\mathrm{S}$, et al. A novel potent vasoconstrictor peptide produced by vascular endothelial cells. Nature 1988;332:411-15.

24 von Lueder TG, Kjekshus $\mathrm{H}$, Edvardsen $\mathrm{T}$, et al. Mechanisms of elevated plasma endothelin-1 in chf: Congestion increases pulmonary synthesis and secretion of endothelin-1. Cardiovasc Res 2004;63:41-50.

25 Beghetti M, Black SM, Fineman JR. Endothelin-1 in congenital heart disease. Pediatr Res 2005:57:16r-20r.

26 Kinugawa T, Kato M, Ogino $\mathrm{K}$, et al. Plasma endothelin-1 levels and clinical correlates in patients with chronic heart failure. J Card Fail 2003;9:318-24

27 Pacher $\mathrm{R}$, Stanek B, Hulsmann $\mathrm{M}$, et al. Prognostic impact of big endothelinplasma concentrations compared with invasive hemodynamic evaluation in severe heart failure. J Am Coll Cardiol 1996;27:633-41.

28 Tatani SB, Carvalho AC, Andriolo A, et al. Echocardiographic parameters and brain natriuretic peptide in patients after surgical repair of tetralogy of fallot. Echocardiography (Mount Kisco, N.Y.) 2010;27:442-7.

29 Koch AM, Zink S, Glockler M, et al. Plasma levels of b-type natriuretic peptide in patients with tetralogy of fallot after surgical repair. Int J Cardiol 2010;143:130-4.

30 Babu-Narayan SV, Uebing A, Davlouros PA, et al. Randomised trial of ramipril in repaired tetralogy of fallot and pulmonary regurgitation: The appropriate study (ace inhibitors for potential prevention of the deleterious effects of pulmonary regurgitation in adults with repaired tetralogy of fallot). Int I Cardiol 2012;154:299-305 